\title{
Efecto del tamaño de trampas adhesivas amarillas para el muestreo de Frankliniella occidentalis en pimiento (Capsicum annum) en invernadero
}

\author{
Paola Carrizo ${ }^{1}$ \\ Cátedra de Zoología Agrícola. Facultad de Agronomía. Universidad de Buenos Aires. Av. San Martín 4453. \\ C1417DSE. Ciudad Autónoma de Buenos Aires. Argentina.
}

\begin{abstract}
P. Carrizo. 2008. Effects of yellow trap size on sampling efficiency for western flower thrips (Frankliniella occidentalis) from pepper plants (Capsicum annum) grown in greenhouses. Cien. Inv. Agr. 35(2):191-197. Sticky colored traps are used in many colors and sizes to capture flower thrips (Frankliniella occidentalis). Although the effects of different trap colors has been extensively tested, the effects of trap size have not. The goal of this work was to study of the relationship between trap size and sampling efficiency for flower thrips collected from pepper plants under greenhouse conditions. Five different yellow sticky trap sizes ranging from 100 to $750 \mathrm{~cm}^{2}$ were tested over three consecutive seasons. The total number of insects caught per trap was proportional to trap area and perimeter length, whereas the number of insects caught per unit area varied with the trap perimeter/area ratio. The latter measure of capture effectiveness was significantly higher for the smallest trap size. Based on these results, one small trap would be needed every $90 \mathrm{~m}^{2}$ or every $5 \mathrm{~m}^{2}$ to sample insects with 20 and $5 \%$ standard error, respectively. We have demonstrated that optimum trap number varies with trap size, an issue that is not usually considered. In addition, the accepted error, a main parameter for sampling protocols that is not defined for traps of different sizes, has been established for five different trap sizes.
\end{abstract}

Key words: Greenhouse crops, insect pests, pepper, thrips, sticky traps, yellow trap size.

\section{Introducción}

De acuerdo con trabajos clásicos la captura en trampas adhesivas no se incrementa en proporción con su tamaño, siendo más eficientes las trampas pequeñas que las grandes (Staples y Allington, 1959; Heathcote, 1957). Según Chen et al. (2006) la captura se relaciona asimismo con el perímetro de la trampa, más que con su superficie. Resultados similares obtuvo Kirk (1987). Estos resultados difieren de lo informado por Carrizo y Benítez (2002) en trampas adhesivas amarillas y Vernon y Gillespie (1995) en trampas de diversos colores y diferentes tamaños.

Actualmente se comercializan trampas de más

Recibido 04 octubre 2007. Aceptado 01 abril 2008

'Dirigir correspondencia a P. Carrizo: pcarrizo@agro.uba.ar de $500 \mathrm{~cm}^{2}$, aunque pueden resultar imprácticas por el tiempo involucrado en su revisión (Shipp, 1995). No obstante, se recomiendan trampas de color amarillo y azul de diversos tamaños sin que necesariametne existan antecedentes que fundamenten esta recomendación. La tendencia general es hacia las trampas de pequeño tamaño, sin una estandarización del número de unidades por superficie (Koppert, 2007; Buxton y Finlay, 1993; Jacobson, 1993; Wardlow et al., 1993).

Si bien respecto del color de las trampas, la eficiencia de captura relativa ha sido probada para varios grupos de insectos (Yudin et al., 1987), existen escasos ensayos comparativos respecto del efecto del tamaño de las trampas en la eficienca de captura (Chen et al., 2006; Carrizo y Benítez, 2002; Vernon y Gillespie, 1995; Kirk, 1987). Por otra parte, con la excepción de los resultados comunicados por Carrizo et al. (2001), para trampas azules de 
$100 \mathrm{~cm}^{2}$ no existen referencias sobre el número de unidades necesarias para un dado nivel de error. Tampoco en los ensayos comparativos para tamaños de trampa se ha considerado la influencia de la superficie de captura de la trampa, en el número de muestras por trampas requeridas.

A nivel experimental, existen considerables discrepancias en diferentes cultivos. Sin embargo, se recomienda comercialmente igual número de trampas por superficie para todos los colores y tamaños de trampa y para diferentes grupos de insectos (Koppert, 2007).

Se debería esperar que el tamaño afecte la eficiencia de captura de las trampas, y afecte el número de unidades necesarias para realizar eficientemente los muestreos. El objetivo del presente trabajo fue estudiar la relación entre el tamaño de trampas adhesivas amarillas y el número necesario para el muestreo del trips de California en pimiento.

\section{Materiales y métodos}

\section{Plantas de pimiento}

Los ensayos se realizaron en invernaderos $\left(800 \mathrm{~m}^{2}\right)$ de pimiento (Capsicum annum) de producción comercial sin aplicación de plaguicidas ubicados en el cinturón hortícola de los alrededores de La Plata (345' lat. S, $57^{\circ} 55^{\prime}$ long. O), en 2003, 2004 y 2005. Las plantas se transplantaron a 1 x $0,30-0,40$ $\mathrm{m}$, con fertirrigación por goteo, se podaron y condujeron en espaldera de alambre. Se muestrearon las flores para verificar la identidad de las especies de trips presentes (Mound y Kibby, 1998; Mound y Marullo, 1996).

\section{Trampas de insectos}

Se utilizaron trampas adhesivas amarillas de $10 \times 10 \mathrm{~cm}\left(100 \mathrm{~cm}^{2}\right), 10 \times 15 \mathrm{~cm}\left(150 \mathrm{~cm}^{2}\right)$, $15 \times 20 \mathrm{~cm}\left(300 \mathrm{~cm}^{2}\right), 20 \times 25 \mathrm{~cm}\left(500 \mathrm{~cm}^{2}\right)$ y $30 \times 25 \mathrm{~cm}\left(750 \mathrm{~cm}^{2}\right)$. Las trampas fueron dispuestas inmediatamente por encima de las plantas en floración que corresponde a la etapa de mayor atracción para trips (Carrizo, 1998; Gillespie y Vernon, 1990). Se formaron cinco grupos de cinco trampas, una de cada tamaño, totalizando 25 puntos de muestreo por cada fecha. La posición relativa de cada tamaño dentro de cada grupo de determinó aleatoriamente. Las trampas permanecieron en el invernadero durante una semana. Para facilitar el recuento de los insectos, se aplicó el adhesivo en un film transparente, adosado a cada trampa (Carrizo, 1998). En el laboratorio se retiró el film y se dispuso sobre una cartulina de color blanco, en la cual se dibujó una grilla de $1 \times 1 \mathrm{~cm}$. Se tomaron muestras desde cada film para verificar la identidad de los trips presentes (Carrizo, 1998; Mound y Kibby, 1998; Mound y Marullo, 1996).

\section{Diseño y análisis estadísticos}

En el invernadero, los tratamientos se asignaron al azar de acuerdo con un diseño de bloques completos al azar, considerando cada hilera como un bloque (Figura 1). Previo a cada análisis, los datos se transformaron a raíz cuadrada (Zar, 1999). Los resultados obtenidos, capturas totales y captura $\mathrm{cm}^{-2}$, en cada una de las 13 fechas (cinco en la primera campaña y cuatro en la segunda y tercera campaña) se sometieron a análisis de varianza (ANDEVA). Los promedios se separaron de acuerdo con la prueba de comparación múltiple de Tukey ( $\mathrm{p} \leq$ 0,05) (Zar, 1999).

Mediante análisis de regresión lineal, se estudió la relación entre las medidas de la trampa y las capturas obtenidas, donde $\mathrm{X}$ fue el perímetro de la trampa o la relación entre el perímetro y la superficie de la trampa, e Y fue la captura total obtenida o captura $\mathrm{cm}^{-2}$ (Zar, 1999).

El tamaño adecuado de muestreo (n, número de trampas) se estimó a partir del error estándar $\left(\sigma_{\mathrm{x}}=\sqrt{ } \sigma^{2} / \mathrm{n}\right)$ para un tamaño de muestra determinado, considerando alternativamente un error estándar igual a 5, 10, 15 y 20\% de la media (Zar, 1999).

\section{Resultados}

Sin excepción, los trips capturados en las flores y en las trampas correspondieron a $F$. occidentalis (Pergande), trips de las flores de California (Mound y Kibby, 1998; Mound y Marullo, 1996). Estos resultados confirman 


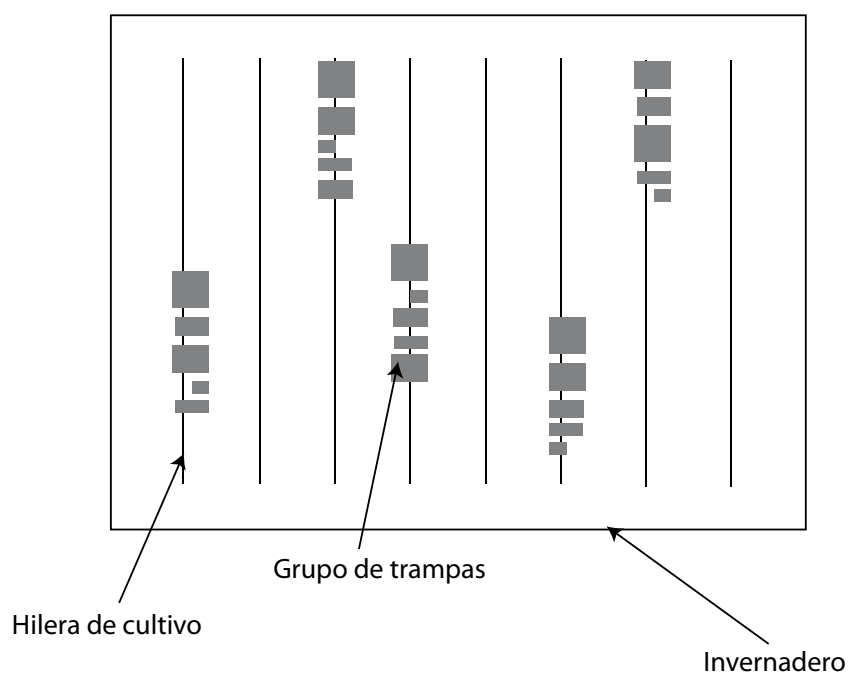

Figura 1. Esquema de la distribución en el invernadero de los grupos de trampas y de los tamaños de trampa dentro de cada grupo en un cultivo de pimiento bajo condiciones de invernadero.

Figure 1. Arrangement of trap sizes within trap groups and the location of these trap groups within the pepper crop growing area under greenhouse condition.

la identificación de esta especie previamente realizada en este cultivo en esta zona goegráfica (Carrizo, 1998).

Según los resultados del ANOVA, hubo un efecto significativo de los tratamientos, tanto para captura total $(\mathrm{p} \leq 0,0001)$ como para captura $\cdot \mathrm{cm}^{-2}(\mathrm{p} \leq 0,0001)$. En relación con la captura total se generaron tres grupos, siguiendo la superficie total de la trampa y en relación con la captura $\cdot \mathrm{cm}^{-2}$ hubo cuatro grupos (Figuras 2a, b). En la misma Figura 2 se graficaron los mejores ajustes obtenidos para la regresión en cada variable (Cuadro 1).
Por lo tanto, las capturas totales respondieron a la superficie total y al perímetro, mientras que la captura por superficie relacionó el perímetro de la trampa y su superficie.

De la aplicación de la fórmula para el número adecuado de muestras, se obtuvieron los resultados de la Figura 3. En la Figura 3a se indican el número de muestras (trampas) necesarias para un determinado nivel de error. El invernadero tuvo $800 \mathrm{~m}^{2}$ de superficie cubierta y de acuerdo con los resultados obtenidos al utilizar cinco unidades de muestreo de una tranpa cada uno, se trabajó con un error levemente superior al $20 \%$. Esto fue

Cuadro 1. Regresión lineal entre el número total de insectos capturados y el número capturado por $\mathrm{cm}^{2}(\mathrm{n}=627)$.

Table 1. Linear regression analysis for total number of insects caught and number caught $\cdot \mathrm{cm}^{2}(n=627)$.

\begin{tabular}{|c|c|c|c|c|c|c|}
\hline Variable dependiente & Variable independiente & $\mathrm{R}^{2}$ & $\mathrm{t}$ & $\mathrm{p}$ & $\mathrm{F}(1 \mathrm{gl})$ & $\mathrm{p}$ \\
\hline Captura total, no. & Superficie & 0,76 & 44,93 & $<0,0001$ & 2018,6 & $<0,0001$ \\
\hline Captura total, no. & Perímetro/superficie & 0,51 & 25,51 & $<0,0001$ & 650,9 & $<0,0001$ \\
\hline Captura total, no. & Perímetro & 0,82 & 54,28 & $<0,0001$ & 2946,2 & $<0,0001$ \\
\hline Captura $\cdot \mathrm{cm}^{-2}$ & Superficie & 0,29 & 54,99 & $<0,0001$ & 3024,2 & $<0,0001$ \\
\hline Captura $\cdot \mathrm{cm}^{-2}$ & Perímetro/superficie & 0,83 & 15,86 & $<0,0001$ & 251,5 & $<0,0001$ \\
\hline Captura $\cdot \mathrm{cm}^{-2}$ & Perímetro & 0,48 & 23,97 & $<0,0001$ & 574,8 & $<0,0001$ \\
\hline
\end{tabular}



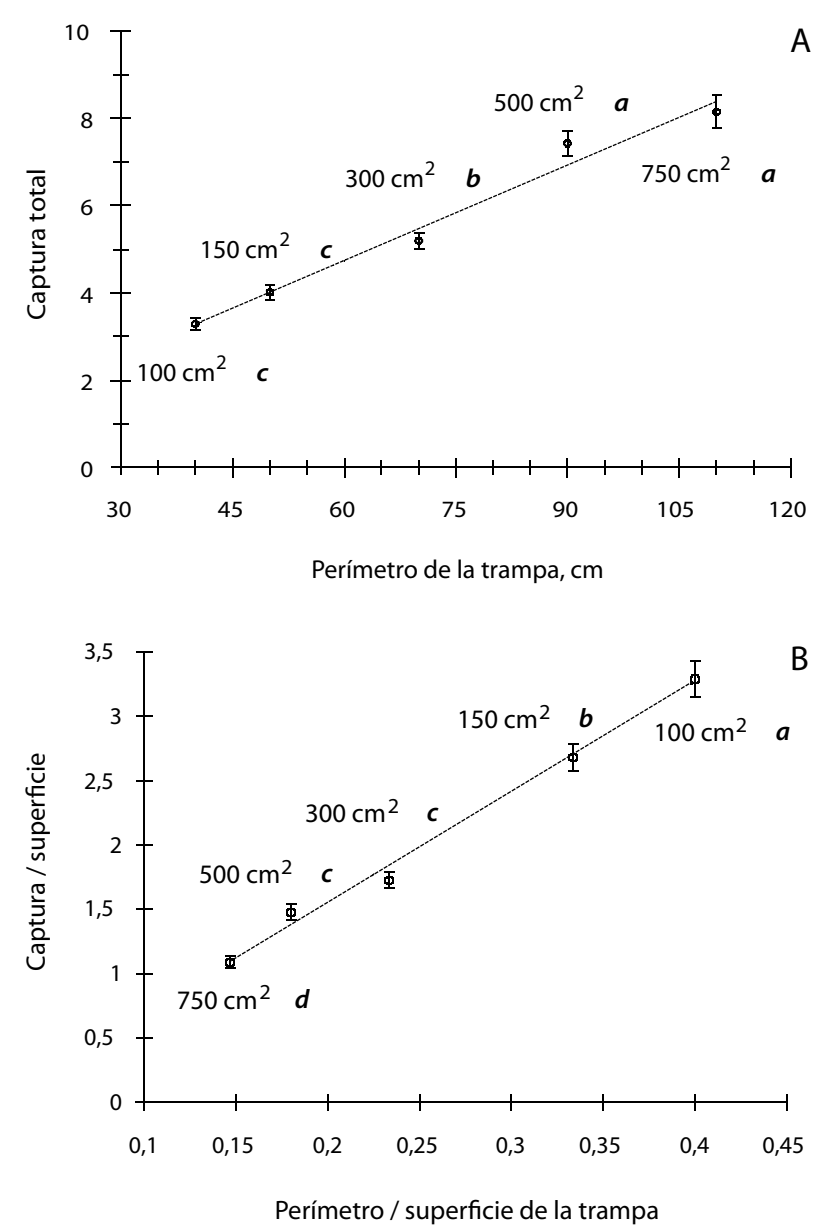

Figura 2. Relación ente el perímetro de la trampa y las capturas totales (A) y relación entre el perimetro/superficie de la trampa y la captura/superficie (B). Promedios seguidos por iguales leras no son significativamente diferentes entre sí de acuerdo con la prueba de comparación múltiple Tukey $(\mathrm{p}=0,05)$. Barras = error estándar.

Figure 2. Relationship between trap perimeter and total number of insects caught (A), and relationship between the ratio of perimeter/area of trap and the number of insects caught per unit area $\left(\mathrm{cm}^{2}\right)(B)$. Means followed by the same lower case letters are not significantly different according to Tukey's tests $(p=0.05)$. Bars $=$ standard error.

ligeramente mejor a lo recomendado, de cinco trampas cada una de $1000 \mathrm{~m}^{2}$, lo que implicaría un error superior al $25 \%$ para cualquier tamaño de trampa (Figura 3a) (Koppert, 2007).

\section{Discusión}

En el presente trabajo se demostró que el número de trampas adecuado para el monitoreo varía con su tamaño, lo cual difiere de las recomendaciones usuales. También se proporciona el error esperado al utilizar éstas trampas, y su cambio a través de las diferentes densidades, un aspecto básico para un protocolo de muestreo, que sin embargo no se menciona para las trampas adhesivas.

Heathcote (1957) utilizando trampas cilíndricas negras para capturar pulgones (Aphidiidae: Hemiptera) probó que las capturas no aumentaron de modo proporcional con el tamaño, por lo cual las trampas más pequeñas fueron más eficientes por unidad de superficie. Por su parte, Staples y Allington (1959) llegaron a una conclusión similar, aunque para la captura de un ácaro (Eriophidae: Acari). 
En ambos casos, dichas trampas funcionaron exclusivamente como interceptoras del viento, ya que superficies negras no atraen pulgones $y$ los ácaros no vuelan (Yudin et al., 1987). Por lo tanto, y a diferencia del presente trabajo, el efecto de preferencia por el color no estuvo involucrado.

Por su parte, Chen et al., (2006) observó capturas proporcionales al perímetro de la trampa cuando éstas mantienen una superficie constante.
Algo similar ocurrió según Kirk (1987), quien informó que en sus trampas circulares podía apreciarse visualmente un agrupamiento de los trips en sus bordes. Carrizo (2000) verificó éste efecto en trampas rectangulares amarillas y azules de $130 \mathrm{~cm}^{2}$. Esto se podría relacionar con el comportamiento de los trips, los que mostrarían una tendencia a dirigirse hacia las áreas con mayor contraste de color (Vernon y Gillespie, 1995).
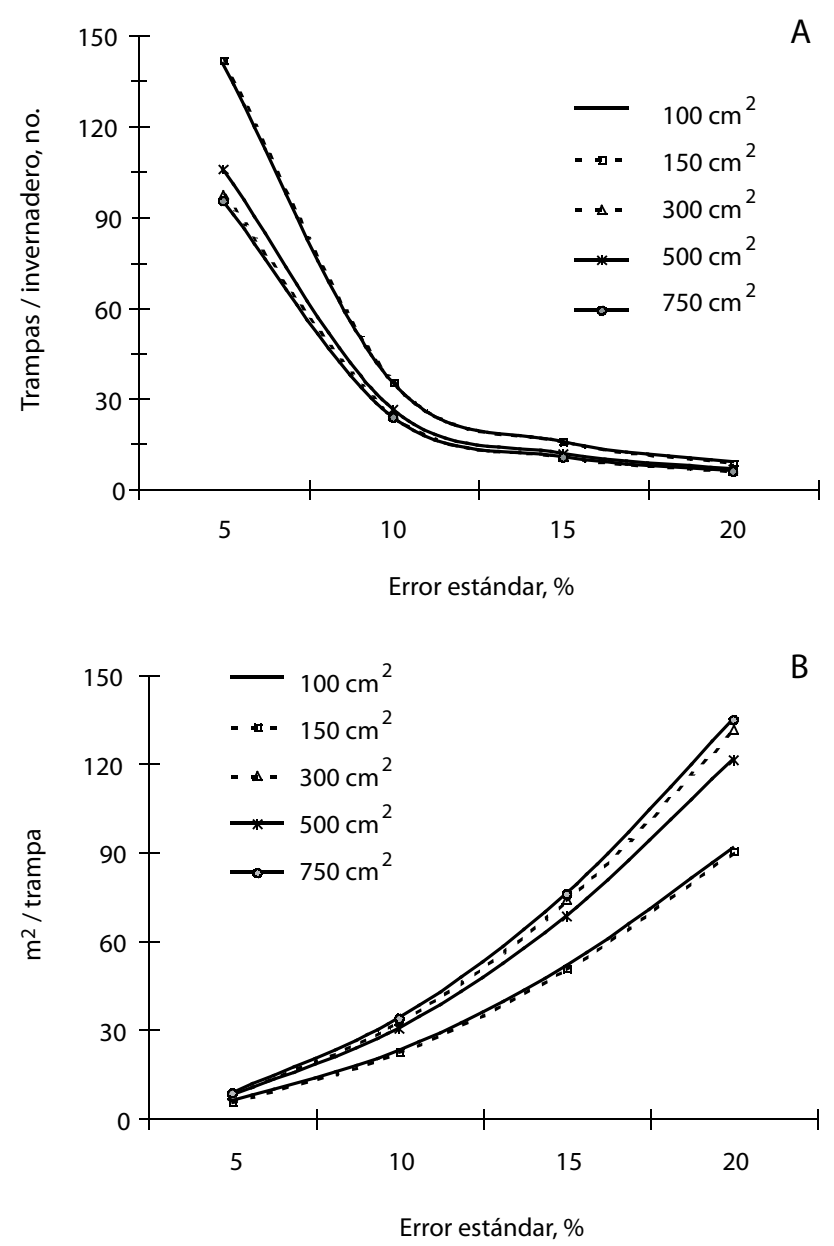

Figura 3. Relación entre el error de muestreo y el número de trampas y tamaño de trampas y la distribución según tamaño de las trampas. A. Número de trampas requerido por invernadero para diferentes niveles de error estándar. B. Area por trampas $\left(\mathrm{m}^{2} \cdot\right.$ trampa $\left.^{-1}\right)$ requerido para diferentes niveles de error estándar. Estimaciones por cada tamaño de trampa para errores estándares entre entre 5 y $20 \%$.

Figure 3. Relationships between sampling error and numbers of traps and trap distribution for different trap sizes. A. Number of traps per greenhouse required for different standard error levels. B. Area covered per trap $\left(\mathrm{m}^{2} \cdot \mathrm{trap}^{-1}\right)$ required for different standard error levels. Estimations for every trap size and for standard errors between 5 and $20 \%$ are shown. 
La relación entre el perímetro y la superficie se redujo a medida que su superficie aumentó. Por lo tanto, la reducción de ésta relación con el incremento de su tamaño sería más evidente para una forma de lados rectos, dado que para la misma superficie total, la relación perímetro: superficie fue mayor. Dado que las trampas comerciales son cuadradas o rectangulares éste efecto sería aún más marcado en ellas (Koppert, 2007).

Por otra parte, Vernon y Gillespie (1995) obtuvieron sus conclusiones a partir de un único ensayo en campo, lo que resulta insuficiente para fines de extrapolación. Sobre la base de resultados previos la diferencia se podría deber a la densidad de trips, considerablemente menor en aquellos ensayos (Carrizo y Benítez, 2002).

La tendencia de las curvas indicadas en la Figura 3a,b muestran claramente que el tamaño de trampa influyó sobre el número de unidades de trampas adecuadas. Sin embargo, uno de los fabricantes más conocidos de trampas adhesivas de colores (Koppert, 2007) recomienda, independientemente del cultivo y de las plagas 5 trampas amarillas ó azules cada $1.000 \mathrm{~m}^{2} \mathrm{de}$ invernadero sean estas de $25 \times 10 \mathrm{~cm}$ o de 25 $\mathrm{x} 20 \mathrm{~cm}$.

La Figura 3a, tiene la forma de la presentación más convencional, donde para un determinado nivel de error, se puede hallar el número de muestras (número de trampas) necesarias. La Figura $3 b$ se relaciona con la posible extrapolación de los resultados presentados. Si bien el modo más adecuado de tomar una muestra es al azar (Southwood, 1994), ese planteo en producción comercial puede resultar engorroso. La tendencia es disponer las trampas en forma sistemática, ya que ello facilita su manejo; por ello, en la Figura 3b se reflejó una indicación de tipo práctica sobre el espaciamiento máximo aceptable entre unidades de muestreo (trampas) a fin de no sobrepasar un determinado error en los recuentos.

\section{Resumen}

Las trampas adhesivas cromáticas son comercializadas y recomendadas en diversos colores y tamaños. Si bien ha sido verificada la eficiencia relativa para varios grupos de insectos respecto del color, no se hallaron referencias respecto a su tamaño. El objetivo de este trabajo fue estudiar la relación entre el tamaño de trampa y su eficacia relativa en la captura de Frankliniella occidentalis en pimiento en invernadero. Se utilizaron trampas amarillas de cinco tamaños, entre 100 y $750 \mathrm{~cm}^{2}$ en un diseño de bloques completos al azar (DBCA, $\mathrm{n}=5)$ y durante 3 campañas consecutivas (13 fechas = 5, 4 y 4). Se utilizaron ANOVA, Tukey y Regresión lineal $($ alfa $=0,05)$. Se determinó el tamaño adecuado de muestra (número de trampas) para los diversos tamaños y para un error estándar entre el 5 y el $20 \%$. Las capturas totales respondieron a la superficie total y al perímetro de la trampa, mientras que las capturas por unidad de superficie $\left(\mathrm{cm}^{2}\right)$, a la relación entre el perímetro y su superficie. La captura por unidad de superficie fue significativamente mayor en las trampas pequeñas. Para un error del $20 \%$, debería colocarse 1 trampa pequeña cada $90 \mathrm{~m}^{2}$, o cada $5 \mathrm{~m}^{2}$ para un error del 5\%. En el presente trabajo se demostró que el número de trampas adecuado para el monitoreo varía con su tamaño, lo cual difiere de las recomendaciones usuales. También se proporciona el error esperado al utilizar éstas trampas, y su cambio a través de las diferentes densidades, un aspecto básico para un protocolo de muestreo, que sin embargo no se menciona para las trampas adhesivas.

Palabras clave: Pestes, pimiento, plagas, tamaño de trampa, trampas adhesivas amarillas, trips.

\section{Agradecimientos}

Se agradece a los productores del cinturón hortícola de La Plata, Argentina, por la colaboración recibida para la ejecución de estos ensayos. Este trabajo fue parcialmente financiado por el proyecto AG-062 (2004-2007), Universidad de Buenos Aires, Argentina.

\section{Literatura citada}

Buxton, J.H. y R. Finlay. 1993. Integrated pest management in Ayr Chrysanthemum. Bulletin OILB-SROP 16:33-41.

Carrizo, P. 1998. Eficiencia de captura en trampas 
sobre Frankliniella occidentalis en cultivos de pimiento en invernáculo y en malezas en el Gran La Plata. Revista de la Facultad de Agrononomía, U.N.L.P. (Argentina) 103:1-10.

Carrizo, P. 2000. Mínima superficie de recuento en trampas adhesivas para trips y mosquitas blancas en pepino de invernadero. Acta Entomológica Chilena 24:45-49.

Carrizo, P. y R. Klasman. 2001. Determinación del número óptimo de trampas adhesivas azules para el monitoreo de Frankliniella occidentalis (Tysanoptera: Thripidae) en Dianthus caryophyllus en invernadero. Folia Entomológica Mexicana 40:389-396.

Carrizo P. y D. Benitez. 2002. Frankliniella occidentalis. Captura con trampas adhesivas amarillas. Revista de la Facultad de Ciencias Agrarias de Cuyo (Argentina) 35:87-91.

Chen, T., C. Chu y T.J. Henneberry. 2006. Influence of trap configuration and size on Frankliniella occidentalis (Tysanoptera: Thripidae) captures. Southwestern Entomologist 31:157-162.

Gillespie, D.R. y S.S. Vernon. 1990. Trap catch of western flower thrips is affected by colour and height of sticky traps in mature greenhouse cucumber crops. Journal of Economic Entomology 83:971-975.

Heathcote, G.D. 1957. The optimum size of sticky aphid traps. Plant Pathology 6:104-107.

Jacobson, R.J. 1993. Integrated pest management in spring bedding plants: a successful package for commercial crops. Bulletin OILB-SROP 16:105-112.

Kirk, W.D.J. 1987. Effects of trap size and scent on catches of Thrips imaginis Bagnall. Journal of the Australian Entomological Society 26:299302.
Koppert. 2007. Koppert Biological Systems. Guidelines and Pest Management Products. http://www.koppert.nl/ (Consultado: Febrero 2007).

Mound, L.A. y G. Kibby. 1998. Thysanoptera. An identification Guide. 2nd ed. CAB Int. UK. 70 pp.

Mound, L.A. y R. Marullo. 1996. The Thrips of Central and South America. An Introduction (Insecta: Thysanoptera). Memoirs on Entomology, International. Vol. 6. Assoc. Publ. Florida, USA. 487 pp.

Southwood, T.R.E. 1994. Ecological Methods. Chapman and Hall. London 3rd. 524 pp.

Shipp, J.L. 1995. Monitoring of western flower thrips on glasshouse and vegetables crops. p: 547-555. In: Thrips Biology and Management. B.L. Parker (ed.). Plenum Presss, NY, USA.

Staples, R. y W.B. Allington. 1959. The efficiency of sticky traps in sampling epidemic populations of the Eriophyd mite Aceria tulipae (K.) vector of wheat streak mosaic virus. Annals of the Entomological Society of America 52:159-164.

Vernon, R.S. y D.R. Gillespie. 1995. Influence of trap shape, size, and background colour on captures of Frankliniella occidentalis in a cucumber greenhouse. Journal of Economic Entomology 88:288-293.

Wardlow L.R., P.J. Davies y W. Brough. 1993. Integrated pest management techniques in protected ornamental plants. Bulletin OILBSROP 16:149-157.

Yudin, L.S., W.C. Mitchell y J.J. Cho. 1987. Colour preference of thrips with reference to aphids and leafminers in hawaiian lettuce farms. Journal of Economic Entomology 80:51-55.

Zar, J.H. 1999. Biostatistical Analysis. 4th ed. Prentice-Hall, Inc. Simon and Schuster/A viacom Co. New Jersey. USA. 663 pp. 